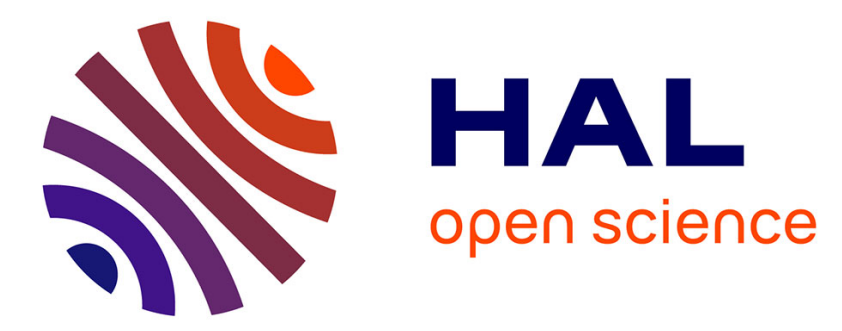

\title{
Buckling resistance of solid shell bubbles under ultrasound
}

Philippe Marmottant, Ayache Bouakaz, Nico de Jong, Catherine Quilliet

\section{To cite this version:}

Philippe Marmottant, Ayache Bouakaz, Nico de Jong, Catherine Quilliet. Buckling resistance of solid shell bubbles under ultrasound. Journal of the Acoustical Society of America, 2011, 129 (3), pp.1231. 10.1121/1.3543943 . hal-00579264

\section{HAL Id: hal-00579264 https://hal.science/hal-00579264}

Submitted on 23 Mar 2011

HAL is a multi-disciplinary open access archive for the deposit and dissemination of scientific research documents, whether they are published or not. The documents may come from teaching and research institutions in France or abroad, or from public or private research centers.
L'archive ouverte pluridisciplinaire HAL, est destinée au dépôt et à la diffusion de documents scientifiques de niveau recherche, publiés ou non, émanant des établissements d'enseignement et de recherche français ou étrangers, des laboratoires publics ou privés. 


\title{
Buckling resistance of solid shell bubbles under ultrasound
}

\author{
Philippe Marmottant \\ Laboratoire de Spectrométrie Physique, CNRS-Université de Grenoble, 140 av de la Physique, 38402 Saint Martin \\ d'Hères, France ${ }^{\text {a) }}$ \\ Ayache Bouakaz \\ UMR INSERM U930 CNRS ERL 3106, Université François Rabelais, 2, Boulevard Tonnellé, 37044 Tours, \\ France \\ Nico de Jong \\ Department of Experimental Echocardiography, P.O. Box 2040, 3000CA Rotterdam, The Netherlands ${ }^{\mathrm{b})}$ \\ Catherine Quilliet \\ Laboratoire de Spectrométrie Physique, CNRS -Université de Grenoble, 140 av de la Physique, 38402 Saint Martin \\ d'Hères, France
}

(Dated: December 15, 2010)

\begin{abstract}
Thin solid shell contrast agents bubbles are expected to undergo different volume oscillating behaviors when the acoustic power is increased: small oscillations when the shell remains spherical, and large oscillations when the shell buckles. Contrary to bubbles covered with thin lipidic monolayers that buckle as soon as compressed, solid shell bubbles resist compression, making the buckling transition abrupt. Numerical simulations that explicitly incorporate a shell bending modulus give the critical buckling pressure and post-buckling shape, and show the appearance of a finite number of wrinkles. These findings are incorporated in a model based on the concept of effective surface tension. This model compares favorably to experiments when adjusting two main parameters: the buckling tension and the rupture shell tension. The buckling tension provides a direct estimation of the acoustic pressure threshold at which buckling occurs.
\end{abstract}

PACS numbers: 43.25.Yw Nonlinear acoustics of bubbly liquids, 43.35.Ei Acoustic cavitation in liquids, 46.32.+x Static buckling and instability

\section{INTRODUCTION}

Bubbles act as powerful contrast agents in ultrasound echography ${ }^{1}$. Current models for coated contrast agents bubbles incorporate the response of the coating $^{2-6}$. These models explore small amplitude vibration, with a linear visco-elastic response from the spherical coating, subjected to alternating compressions and tensions because of the radius oscillation. However, the large amplitude vibration regime is of importance for cases where a non-linear response of contrast agents is wished in order to discriminate them from the surrounding tissue. In addition to non-linear elasticity ${ }^{7}$, two processes can lead to a strong non-linear response: buckling ${ }^{8}$ or destruction of the coating ${ }^{9,10}$.

Buckling was observed in the specific case of lipidic contrast agents, coated with a single layer (monolayer) of lipid molecules sitting at the bubble interface. This coating can lead to peculiar oscillations, named compressiononly ${ }^{11,12}$, because only compression is significant while hardly no expansion occurs, the reason being that lipid monolayer, one molecule thick, does not undergo in-plane compression. The bending modulus of the monolayer is

\footnotetext{
a) Electronic address: Philippe.Marmottant@ujf-grenoble.fr

b) Also at Physics of Fluids Group, Department of Science and Technology, University of Twente, P.O. Box 2177500 AE Enschede, The Netherlands
}

very small, so it is energetically more favorable to bend the membrane that to compress it.

In the present manuscript, we focus on a different kind of contrast agents: bubbles coated with solid membranes, whose thickness is large in front of molecular components. These coatings are made of proteins (albumin for instance) and polymers ${ }^{13}$. Because of their thickness, such coatings can be described using continuum theories. Contrary to lipid monolayers, spherical solid shells ${ }^{14}$ sustain more easily in-plane compressions, which stabilizes bubbles against dissolution ${ }^{15}$. This holds for limited compressions though: contrast agents also undergo buckling if the compressive constraint is large enough. Contrary to lipidic agents that develop very tiny wrinkles around a globally spherical shape ${ }^{11}$, solid shell bubbles display a globally non-spherical shape.

Here we explore the periodic buckling and unbuckling of these solid shells, thus extending the solid shell models designed to treat spherical oscillations with a limited amplitude $^{2,8}$. The appearance of wrinkles was reported on bubbles undergoing dissolution ${ }^{16}$, here they occur after buckling in each sound cycle. The shape of the wrinkles is modeled with 3D numerical simulation, and retrieves the experimental observations thanks to the use of a bending modulus under the assumption of quasistatic deformation, while membrane computations ${ }^{17}$ usually neglect this bending to obtain the onset of buckling in a dynamical situation.

We also address the destruction of bubble shells. Destruction phenomena have been applied successfully to 
image tissue perfusion and perfusion defects, leading to effective techniques for myocardial perfusion imaging after intravenous injection. The destruction of gas microbubbles is the basis of triggered or intermittent imaging methods ${ }^{18}$. Microbubble destruction is found in the destruction-replenishment methods to estimate blood perfusion ${ }^{19}$. Extensive research has also been carried out based on the bubble destruction mechanism toward drug and gene delivery ${ }^{20,21}$. The destruction of coated microspheres showed to be a promising method for the delivery of drugs and other active agents to various organs in the body ${ }^{22-24}$. These investigations opened new perspectives for therapeutic applications but the use of contrast gas microspheres toward such applications brings the need for a thorough understanding of the processes involved in their destruction. In order to select the ultrasound parameters and the scanning strategies for an optimal use in drug and gene delivery, understanding the destruction process is essential.

The manuscript is organized as follows: section II presents detailed numerical simulations of the buckling of initially spherical shells, in order to explicit the inner pressure during compression, as well as the shell shape. Based on these findings, an analytical model for pulsation of the bubble is introduced in section III. The main feature of the model, an abrupt appearance of buckling or break-up at two distinct thresholds in acoustic pressure, is presented in section IV. Finally section V compares these findings with experimental data.

\section{BUCKLING OF A SPHERICAL SHELL: INNER PRESSURE DURING DEFLATION}

\section{A. Numerical methods for the quasi-static simulation of an elastic surface with bending and compression moduli}

To understand the effect of an applied pressure on a solid shell we model it by a closed surface, initially spherical. We present in this subsection numerical simulations of its 3D shape, when the enclosed volume $V$ decreases ("deflation"). At the end of this subsection and in Appendix $\mathrm{A}$ we present the link between the $2 \mathrm{D}$ parameters of the numerical surface and the 3D parameters of thin shells.

The deformation energy of an homogeneous elastic surface comprises a bending contribution, with a bending constant $\kappa$, and an in-plane contribution for which, in a linear approximation, only two parameters are required, e.g. the compression (or stretch) modulus $\chi_{2 D}$, and the Poisson ratio $\nu_{2 D}$.

For a given volume, the numerical shape is found by minimizing the integral of the surface energy that writes $^{25}$ :

$$
\begin{aligned}
E_{e l}=\int_{S}\left\{\frac{1}{2} \kappa\left(c_{1}+c_{2}-2 c_{0}\right)^{2}\right. & -(1-\nu) \kappa\left(c_{1}-c_{0}\right)\left(c_{2}-c_{0}\right) \\
& \left.+\frac{1}{2} \epsilon_{i j} K_{i j k l} \epsilon_{k l}\right\} d S
\end{aligned}
$$

where the first and second term in the integrand reflect the bending energy: $c_{1}$ and $c_{2}$ are the principal curvature, $c_{0}=1 / R_{0}$ the initial curvature with $R_{0}=\left(3 V_{0} / 4 \pi\right)^{1 / 3}$ the radius of the initial unstrained sphere, and $V_{0}$ its unstrained volume. The first term is associated with total curvature, while the second is associated Gaussian curvature ${ }^{25}$. This second term was usually neglected in simulation of shells, but recent work carried out by the authors pointed out that it does not vanish for geometries with initial spontaneous curvatures, which is the case here. The third term in the integrand is the in-plane Hookean deformation energy, with $\epsilon_{k l}$ is the in-plane Cauchy-Green strain tensor, and the nonzero terms of the two-dimensional elasticity tensor are $K_{i i i i}=\frac{1}{\nu_{2 D}} K_{i i j j}=\frac{1}{1-\nu_{2} D} K_{i j i j}=\frac{2}{1+\nu_{2 D}} \chi_{2 D}$.

A detailed presentation of the numerical method can be found $\mathrm{in}^{26}$. We recall it here briefly. The surface is discretized in a 2D randomized triangular mesh (made of 4764 nodes) fine enough in front of the topographical changes; then the volume is decreased by small steps (with a relative volume decrease of $d V / V_{0}=6.36 \times 10^{-3}$ ), while at each step the shape is evolved, using the finite element freeware Surface Evolver ${ }^{27}$, until a minimization of its energy (given by Eq. 1) is reached. Simulations showed that the spherical surface deforms with different shapes during the deflation, that depend on two nondimensional parameters: the relative volume variation $\frac{\Delta V}{V}=\frac{V-V_{0}}{V_{0}}$ and the Föppl-von Kárman parameter ${ }^{28}$ $\gamma=\frac{2\left(1-\nu_{2 D}\right) \chi_{2 D} R^{2}}{\kappa}$.

Using these simulations in order to interpret experiments requires to make the link between the 2D parameters of the model surface (namely $\kappa, \chi_{2 D}$ and $\nu_{2 D}$ ) and the 3D parameters of the shell, which are the shell thickness $d$ and the bulk shear modulus $G$, assuming the material as incompressible $(\nu=1 / 2)$.

The bending modulus $\kappa$ can be expressed through the integration of the local compression or elongation of the incompressible material when the sheet bends, leading to $^{29}$ :

$$
\kappa=\frac{1}{3} G d^{3}
$$

The general expression for the compression modulus $\chi_{2 D}$ is displayed in Appendix A. For an incompressible material, it leads to:

$$
\chi_{2 D}=3 G d .
$$

We may now understand why the bending of a very thin monolayer is energetically favored compared to compression. One can show that the ratio between outof-plane (bending) energy $E_{b}$ and in-plane compression energy $E_{s}$ in a typical deformation ${ }^{25,29}$ is of the order $E_{b} / E_{s} \sim \kappa / \chi_{2 D} R^{2}$, revealing the key parameter of the system, the non-dimensional Föppl-von Kárman parameter $\gamma=\frac{\chi_{2 D} R^{2}}{\kappa}$, see erratum of ${ }^{26}$. Eq. 2 and 3 lead to

$$
\gamma=\frac{9}{(d / R)^{2}}
$$

meaning that $\gamma^{-1 / 2}$ simply reflects the relative shell thickness $d / R$. This relation explains why a bending 
deformation is energetically more favorable than a compression for thin shells: $E_{b} / E_{s}$ becomes very small when $d / R$ decreases.

For the deformations studied here, Poisson ratios are equal in 2D and 3D (see Appendix A), so that $\nu_{2 D}=\nu=$ $1 / 2$.

\section{B. Deformation modes and the inner pressure}

The results from the simulations are now described. Several computations are performed for shell with different relative thickness, that are imposed through the numerical 2D factor $\gamma$. The typical behavior of a shell under deflation is the following. For small deflations the surface keeps a spherical shape (see fig. 1a). For higher volume deflations, shells undergo a first-order (i.e. discontinuous and abrupt) buckling transition: an inverted spherical cap appears, making an axisymmetrical depression (fig 1b). Then, axisymmetry is broken through polygonal deformation of the depression (fig. 1c), for small enough values of $d / R$ (typically $d / R<0.1$ ). (a)

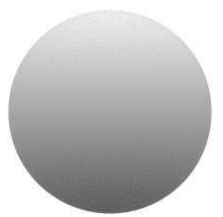

(b)
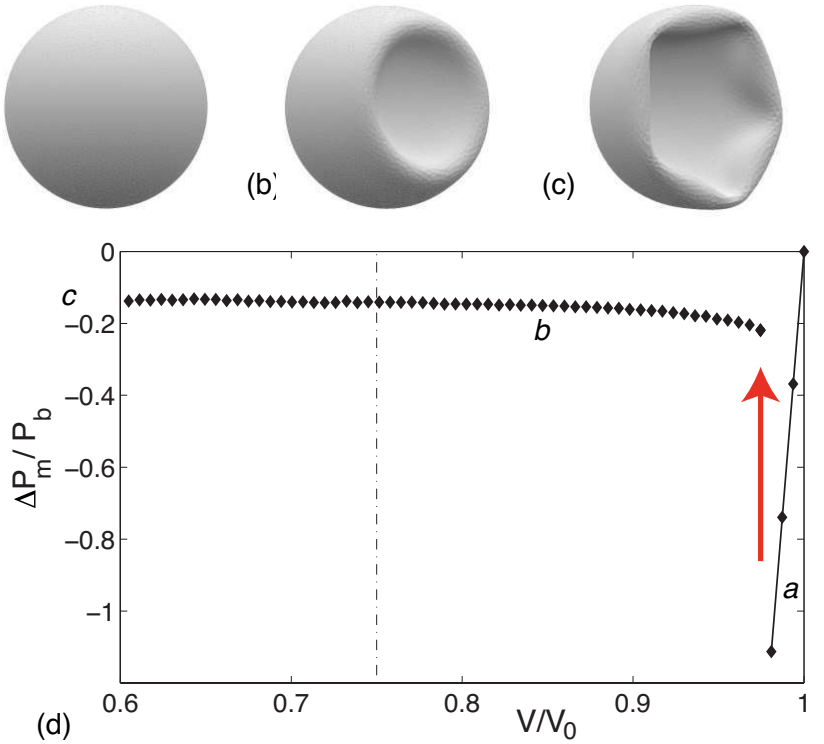

$d E_{e l}=-P_{e x t} d V+P_{i n t} d V$. The pressure is thus inferred by $\Delta P_{m}=\partial E_{e l} / \partial V$. Note that this equality holds because we assumed that no kinetic energy is generated, which we justify here because the mass of the shell is small and assumed to have negligible inertia. We will see later that the mass of fluid around is dominant.

\section{Spherical compression}

In this deformation mode, the pressure varies rapidly and can be predicted analytically.

By considering the energy of a sole in-plane compression (there is no first-order bending contribution in this geometry), $E_{e l}=\frac{1}{2} \chi_{2 D}\left(\frac{A-A_{0}}{A}\right)^{2}$, one gets from $\Delta P_{m}=\partial E_{e l} / \partial V$ the over-pressure inside:

$$
\Delta P_{m}=4 \frac{\chi_{2 D}}{R_{0}} \frac{\Delta R}{R},
$$

with $\Delta R=R-R_{0}$, assuming $\Delta R \ll R_{0}$. In a bulk 3D model, the integrated contribution of the internal elastic stress over the shell thickness lead Church ${ }^{2}$ to obtain:

$$
\Delta P_{m}=12 G \frac{d}{R} \frac{\Delta R}{R_{0}} .
$$

One retrieves the relation $\chi_{2 D}=3 G d$ obtained in Appendix A for flat sheets. Numerical data are consistent with this prediction, showing a linear part in fig. 1 for small amplitude compressions, with the predicted slope.

\section{Buckling}

Under compression, the linear variation into the negative pressure range (meaning a compressive state of the membrane), is limited to a short range. According to ${ }^{29}$ an elastic spherical shell submitted to a smaller pressure inside will become unstable and buckle, and this as soon as the pressure $\Delta P_{m}$ reaches a critical value of

$$
\Delta P_{m}^{\text {buckling }} \sim-3 G\left(\frac{d}{R}\right)^{2},
$$

FIG. 1. Numerical simulation of the quasi-static deflation of an elastic shell (a) $V / V_{0}=1$, (b) 0.85, and (c) 0.48 . (d) The inner pressure when decreasing the volume. Pressure here is nondimensional by the theoretical value for buckling $P_{b}=3 G(d / R)^{2}$. The continuous line is the prediction from Eq. 5, while the dash-dotted line delimits the wrinkled / axisymmetric transition. Here the Föppl-von Kárman parameter is $\gamma=16796$ and $\nu_{2 D}=1 / 2$, corresponding to $d / R=0.0231$.

The numerical pressure (i.e. Lagrange multiplier of the volume) is displayed on figure $1 \mathrm{~d}$. It is the numerical equivalent of the pressure difference $\Delta P_{m}=P_{\text {int }}-P_{\text {ext }}$ between pressure $P_{\text {int }}$ inside of the membrane, and the exterior pressure $P_{\text {ext }}$. This pressure difference follows from the conservation of mechanical energy during a compression: the increase in elastic energy is generated by the work of pressure forces on the membrane, so that this expression is obtained after replacing the Young's modulus by $2 G(1+\nu)=3 G$ for incompressible material. It corresponds to the pressure at which it is more advantageous to create a fold rather than compressing the shell. This order of magnitude, is effectively retrieved in numerical simulations, see figure 1 and 2 .

For instance for Albunex $(\mathrm{R})$ contrast agents made with an albumin shell, $G=88 \mathrm{MPa}, d=15 \mathrm{~nm}, R=1 \mu \mathrm{m}^{8,30}$, meaning $\Delta P_{m}^{\text {buckling }} \simeq 6 \times 10^{4} \mathrm{~Pa}$. This pressure is easily achieved by acoustic means. Solid shell contrast agents are expected to buckle during insonification if their shell is not too thick.

After buckling, the bubble shape suddenly departs from a sphere, the stretching stresses within the shell become much smaller, while bending stresses are localized on the edge of bulge ${ }^{29}$. The pressure difference therefore drops considerably and plateaus with volume variation 
as can be seen on figure 1-d. The corresponding value $\Delta P_{m}^{\text {post-buckling }}$ was measured at its lower absolute value. (Note that for the shells thinner than $\frac{d}{R}<0.0145$, a slight re-increase is observed when $\Delta V / V$ approaches 1$)$. It is displayed on fig 2 as a function of $d / R$, together with the buckling pressure. The pressures are scaled by the bulk shear modulus $G=\frac{1}{9} \sqrt{\chi_{2 D}^{3} / \kappa}$ which imposes the energy scale. The post-buckling pressure is much smaller than the buckling pressure, especially for thin shells. For shells with a thickness smaller than $d / R=10^{-2}$, the ratio of these pressures is 0.1 . This ratio decreases for smaller thickness, meaning that the thinner the shell, the lower the remaining pressure after buckling. Corresponding theoretical calculations can be found in Appendix B.

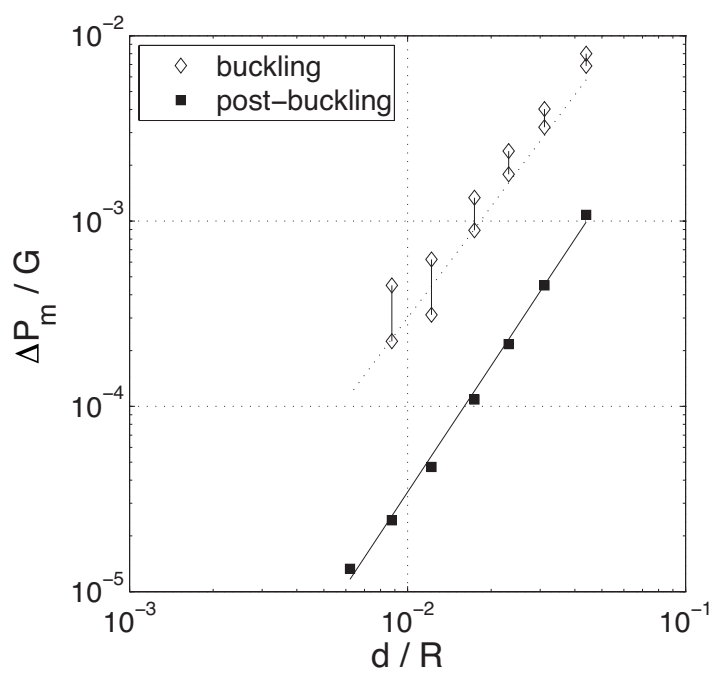

FIG. 2. Simulation results for the post-buckling pressure $\Delta P_{m}^{\text {post-buckling }}$ (squares), evaluated in the plateau region of Fig. 1d, as a function of the relative shell thickness. The continuous line stands for a power-law fit with $\Delta P_{m}^{\text {post-buckling }} / G=1.193 \times(d / R)^{2.27 \pm 0.06}$. The value of the exponent is in total agreement with theoretical predictions of post-buckling pressure derived in Appendix B. For comparison, we also plot the buckling pressure $\Delta P_{m}^{\mathrm{b}}$ from simulations (diamonds giving the range where simulation value is located), and the theoretical prediction (dashed line) from Eq. 7.

\section{Wrinkles}

At larger compression amplitude, the buckled state looses its axi-symmetry, and periodic wrinkles appear in the buckling depression. This post-buckling transition is smooth and cannot be detected on the pressure itself. The number of wrinkles $W$ depends on the relative thickness, see figure 3 . Actually variations of typically \pm 1 (up to \pm 2 for high $W$ ) wrinkles are observed in the course of compression, so the average number of wrinkles has to be considered.

The number of wrinkles approximately follows an evo- lution of the type:

$$
\langle W\rangle \simeq 0.83\left(\frac{d}{R}\right)^{-1 / 2} .
$$

This power law can be retrieved with the consideration that $\sqrt{d R}$ is the typical size for the deformations of a thin shell ${ }^{29}$. On an equator of length proportional to $R$, the number of wrinkles of size $\approx \sqrt{d R}$ scales like $\sqrt{R / d}$.

At re-inflation the number of wrinkles diminishes by the coarsening of neighbour wrinkles.

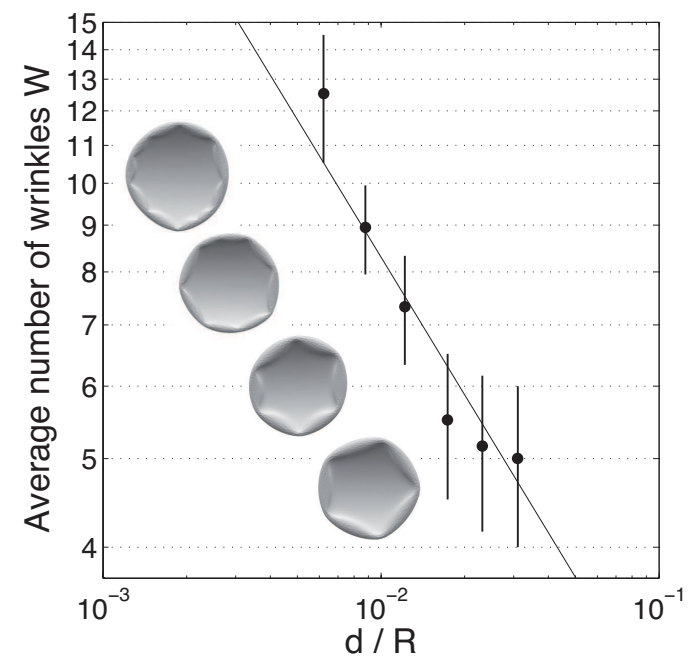

FIG. 3. Simulation of the number of wrinkles hold by the single depression of a deflating elastic spherical surface, as a function of the relative shell thickness for a thin shell of incompressible material. This number is an average for important deflations, i.e. when the size of the depression approaches one hemisphere, and before autocontact ; practically we averaged for $\Delta V / V \in[0.53,0.76]$. Points are dispersed but compatible with the power-law in $(d / R)^{-1 / 2}$ observed $\mathrm{in}^{26}$. Here the line is a fit with Eq. 8. Inserted snapshots are taken from simulations of shells with $10,8,6$ and 5 wrinkles, from left to right.

\section{DYNAMICAL MODEL FOR LARGE AMPLITUDES OSCILLATIONS}

We extrapolate the numerical results obtained for the static case, to describe dynamical oscillations of bubbles. It is reasonable here because the material equilibrates at the speed of sound in the elastic material (typically of several thousands of meters per second) which is very large compared to the bubble velocity $\left(\omega R_{0}\right.$, of the order of meters per second).

\section{A. Effective membrane tension: negative at the onset of buckling and then suddenly vanishing}

The presence of the shell accounts for a pressure difference $\Delta P_{m}$. By analogy with a Laplace pressure, we 
rather express the solid shell contribution as due to an effective membrane tension $\sigma_{m}(R)=R \Delta P_{m} / 2$ such that

$$
\Delta P_{m}=\frac{2 \sigma_{m}(R)}{R} .
$$

The advantage is that membrane tension reflects the average of plane stresses within the membrane, which will be useful when searching for a criteria for shell rupture. This tension depends on the bubble inflation or deflation (here monitored by $R$ with respect to a rest radius $R_{0}$ ), and can even become negative, sign of compression state, when the volume decreases.

Inspired by the numerical simulation of the previous section, we model the solid shelled bubble response under several states: elastic, buckled (axisymmetric or wrinkled), to which we add the ruptured state. The model is summarized on figure 4 . The main difference from the lipid shell model ${ }^{11}$ is that the buckled state occurs after a negative membrane tension, while in the the lipid shell model it occurs as soon as the membrane tension starts to become negative.

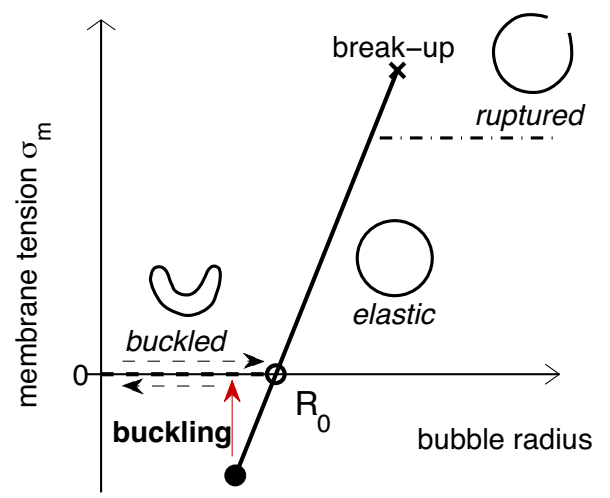

FIG. 4. Model for the shell membrane tension under large volume variation. As soon as the surface tension is negative enough to reach the buckling value (full circle), buckling occurs (large arrow) and the membrane tension vanishes (thick dashed line). The elastic state is recovered when the volume gets back to its rest value (open circle). When the rupture point is reached (cross), membrane tension saturates to the water value (dash dotted).

In the elastic state, the membrane tension varies (thick line on figure 4) as indicated by Eqs. 5 and 9 along:

$$
\sigma_{m}(R)=2 \chi_{2 D} \frac{\Delta R}{R_{0}} .
$$

For the instance of Albunex(R) contrast agents we find $\chi_{2 D}=3 G d=4 \mathrm{~N} / \mathrm{m}$. This linear relation holds here because we are considering small amplitude variations in this regime.

The buckling occurs abruptly when the tension is low enough. As soon as the membrane tension becomes negative enough to reach the critical buckling value

$$
\sigma_{m}^{\text {buckling }} \sim-\frac{1}{6} \chi_{2 D} \frac{d}{R}
$$

the shape becomes unstable and buckles. The critical buckling value we get for Albunex is $\sigma_{m}^{\text {buckling }} \sim-0.01$ $\mathrm{N} / \mathrm{m}$. It corresponds to a relative compression of only $(\Delta R / R)^{\text {buckling }}=-1 / 12 \times d / R_{0}$ (around $0.1 \%$ for $\mathrm{Al}-$ bunex). The membrane tension fades out to a much lower value when the bulge indentation grows, as observed on simulations (figure 2) and computed theoretically in appendix B. Here we therefore assume that membrane tension instantly vanishes at buckling (thick dashes on figure 4).

The membrane stays on this state, as long as it reaches again the stress free point, where the shape becomes spherical again and where the elastic regime is recovered. Note that the notion of a bubble radius disappears in the buckled state with a nonspherical shape: however the bubble volume stays continuous. For the dynamical equations we will keep the radius as a variable, and in the buckling state it will have to be considered as an effective quantity, such as $R_{\text {eff }}=(3 V / 4 \pi)^{1 / 3}$, reflecting the volume and useful to provide an estimate of the average size of the bubble.

On the reverse, if the pressure difference becomes strongly positive the shell becomes tensed, until it reaches rupture. The material of the shell cannot support infinite elongation, it is expected to break apart at a few percent of positive inflation of the shell area, meaning at a critical value $\left(\Delta R / R_{0}\right)^{\text {break-up }}$. Indeed material resistance is usually defined by a maximum deformation amplitude. A positive peak in surface tension and in bubble radius can reach the break-up value: at this point a part of the shell opens and a bare interface of air is exposed to liquid. Being ruptured, the surface tension is therefore bounded to a saturation value $\sigma_{\text {water }}$, the surface tension of the water/gas interface.

Note that the membrane can sustain a positive radius increase $\left(\Delta R / R_{0}\right)^{\text {break-up }}$ independent of the shell thickness, while the maximum radius decrease without buckling is $(\Delta R / R)^{\text {buckling }}=-1 / 12 \times d / R_{0}$ (from Eqs. 11 and 10) and thus proportional to the shell thickness. Considering a harmonic excitation of small amplitude, the bubble response is harmonic (sinusoidal) as well with positive and negative radius excursions of equal amplitudes. Under these conditions, buckling will occur before rupture if $\left|(\Delta R / R)^{\text {buckling }}\right|<(\Delta R / R)^{\text {break-up }}$, or if $d / R<12\left(\Delta R / R_{0}\right)^{\text {break-up }}$. We therefore expect thin solid shells to buckle before they rupture, and the reverse for thick shells.

\section{B. Dynamics of the coated bubble}

We then model the dynamics of the pulsation, following the same line as the model for a lipidic membrane ${ }^{11}$, where a variable tension is introduced in the dynamical evolution for a free bubble. We nonetheless recall here these equations, and add the new modeling for the effective tension.

From the balance of normal stresses at the interface, assuming a polytropic gas law and a assuming modified Rayleigh-Plesset equation for the hydrodynamic pres- 
sure, we obtained:

$$
\begin{aligned}
& \rho_{l}\left(R \ddot{R}+\frac{3}{2} \dot{R}^{2}\right)=\left(P_{0}+\frac{2 \sigma_{m}\left(R_{0}\right)}{R_{0}}\right)\left(\frac{R}{R_{0}}\right)^{-3 \kappa}\left(1-\frac{3 \kappa}{c} \dot{R}\right) \\
& -P_{0}-\frac{2 \sigma_{m}(R, \text { state })}{R}-\frac{4 \mu \dot{R}}{R}-\frac{4 \kappa_{s} \dot{R}}{R^{2}}-P_{a c}(t), \quad \text { (12) }
\end{aligned}
$$

with $R_{0}$ the equilibrium radius of the bubble, with no membrane stress, $P_{0}$ the ambient pressure, $P_{a c}(t)$ the acoustic pressure, and $c$ the velocity of sound in the liquid. Dissipation is introduced with $\mu$ the surrounding liquid viscosity and $\kappa_{s}$ the surface dilatational viscosity from the shell. Note that, contrary to ${ }^{2}$ we neglected the surface tension of the inner and outer solid interfaces (their value been negligible in front of the buckling and rupture tension): all the capillary effects are included in the effective membrane tension.

As developed in the previous section, the membrane tension $\sigma_{m}(R$, state $)$ depends upon the radius and upon the actual state (elastic, buckled, ruptured) with:

$$
\sigma_{m}(R)=\left\{\begin{array}{cc}
2 \chi_{2 D} \frac{\Delta R}{R_{0}} & \text { if elastic, } R_{\text {buckling }}<R<R_{\text {break-up }} \\
0 & \text { if buckled } \\
\sigma_{\text {water }} & \text { if ruptured and } R>R_{\text {ruptured }}
\end{array}\right.
$$

The transition between the elastic and the buckled state occurs as soon as $R<R_{\text {buckling. This change is re- }}$ versible: unbuckling occurs as soon as $R>R_{0}$. The transition to the ruptured state property is not reversible and occurs as soon as $R>R_{\text {break-up }}$.

\section{MODEL RESULTS}

\section{A. Abrupt appearance of compression-only behaviour}

Increasing the acoustic pressure, the bubble oscillates symmetrically in the elastic mode, until it buckles: the bubble oscillation becomes suddenly larger in the compression phase but not in the expansion phase leading to the "compression-only" 12 , see simulation of the dynamical equation on figure 5. A strong positive radius excursion then appears above a critical pressure. In this new state, the bubble oscillates as a free bubble, as the shell is ruptured.

The different behavior are well monitored by plotting the ratio of the positive excursion peak to the negative excursion peak, $\Delta R^{+} / \Delta R^{-}$(see figure 6). Elastic oscillations do not lead to a significant asymmetry $\left(\Delta R^{+} / \Delta R^{-} \simeq 1\right)$, while compression-only modes lead to smaller ratios $\left(\Delta R^{+} / \Delta R^{-}<1\right)$, inversely to the ruptured state: the non-linear behavior then favors positive excursions of the radius, as for standard large pressure Rayleigh-Plesset dynamics of a free bubble $\left(\Delta R^{+} / \Delta R^{-}>1\right)$.

The appearance of compression-only is abrupt above the threshold (for an acoustic amplitude of $1 \mathrm{~Pa}$ in the exemple of figure 6). Again, this in contrast to the model for monolayer lipidic coatings, that did not allow any negative tension, and for which the transition to compressiononly was continuous.

FIG. 5. Bubble response to repeated $2 \mathrm{MHz}$ pulses, with an increasing acoustic pressure: elastic at $0.8 \times 10^{5} \mathrm{~Pa}$, buckled at $1.5 \times 10^{5}$ and ruptured at $2.7 \times 10^{5} \mathrm{~Pa}$. The shell parameters are $R_{0}=1 \mu \mathrm{m}, \chi_{2 D}=1 \mathrm{~N} / \mathrm{m}, \kappa_{s}=7.2 \times 10^{-9} \mathrm{~N}$, while the buckling tension is $\sigma_{m}^{\text {buckling }}=-0.05 \mathrm{~N} / \mathrm{m}$, and the rupture tension is $\sigma_{m}^{\text {break-up }}=0.2 \mathrm{~N} / \mathrm{m}$.
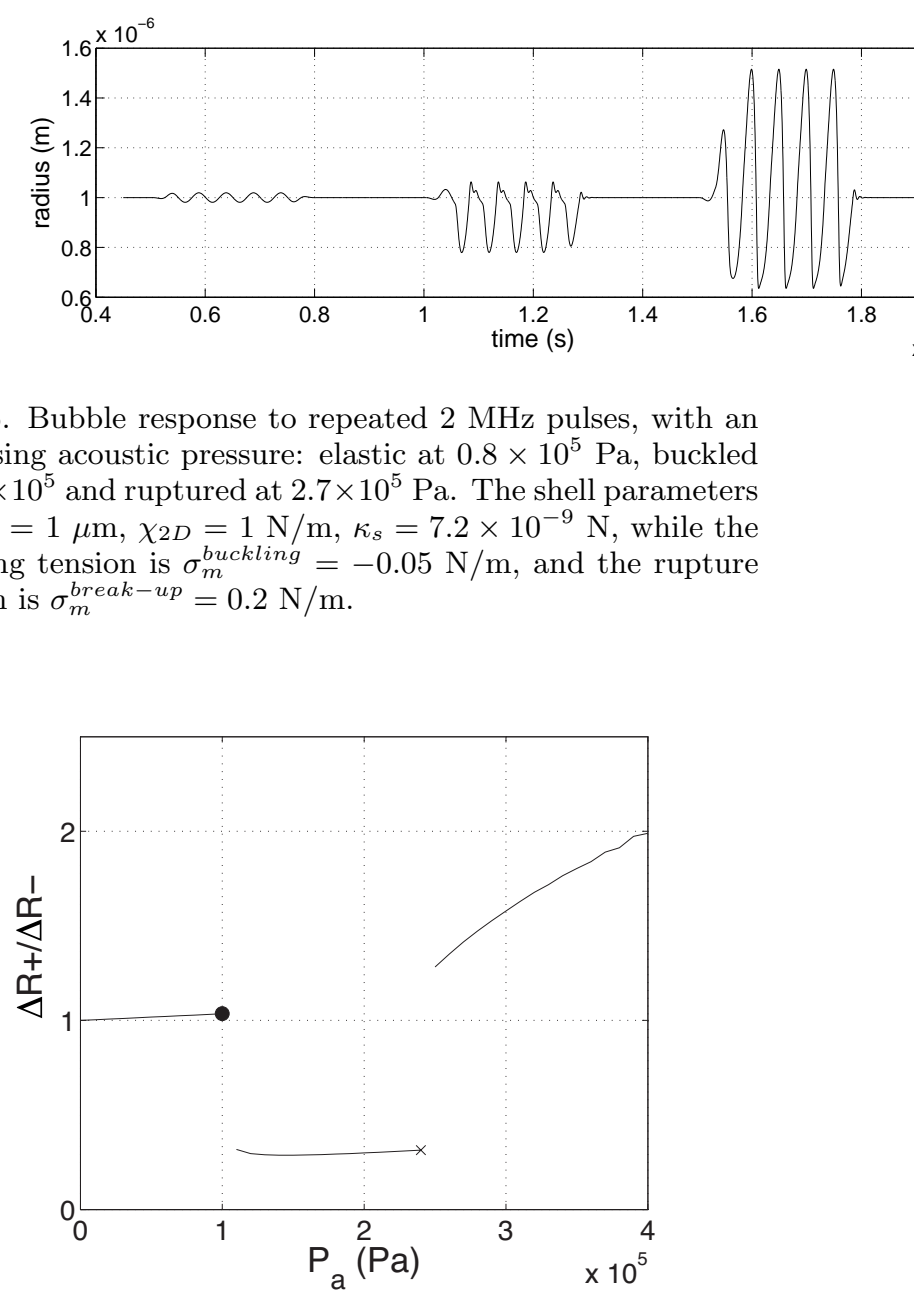

FIG. 6. Effect of an increasing acoustic pressure on the asymmetry of the response. Same shell parameters as in figure 5.

\section{B. Comparison of the dynamic and static value for the buckling pressure}

From the dynamical simulations we can try to evaluate the thresholds for the different regimes and compare them to the static thresholds described in section III.B. The static pressure $2 \sigma_{m}^{\text {buckling }} / R_{0}\left(=1 \times 10^{5} \mathrm{~Pa}\right)$ gives a correct estimate of acoustic pressure at which the elastic state buckles (see figure 6). However the value $2 \sigma_{m}^{\text {break-up }} / R_{0}\left(=4 \times 10^{5} \mathrm{~Pa}\right)$ does not provide a correct estimate for the acoustic pressure at rupture because of the high amplitude oscillations.

Indeed, before buckling the amplitude of oscillation is low so that $P_{l} \simeq P_{0}+P_{a c}(t)$ (see section III.B) and $\Delta P_{m}=P_{g}-P_{l} \simeq-P_{a c}(t)$, meaning that the pressure difference across the membrane is given directly by acoustic pressure. At high amplitudes, the liquid pressure incorporates dynamical terms, and has to be written $P_{l}=P_{0}+P_{a c}(t)+\rho_{l}\left(R \ddot{R}+\frac{3}{2} \dot{R}^{2}\right)+\frac{R}{c} \frac{d P_{g}(t)}{d t} R(t)$ : it is not possible to induce the membrane pressure directly from the acoustic pressure. 


\section{COMPARISON WITH EXPERIMENTS}

\section{A. Phase diagram}

We compare these prediction to the high-speed imaging study ${ }^{9}$ of polymer based shell contrast agents (PB127 contrast agent from Point Biomedical, a bilayer polymer/albumin shell encapsulating a nitrogen bubble). For a given radius, contrast agents exhibit different behavior as a function of pressure, classified in three regimes: (i) non-destruction zone with oscillations, (ii) transient zones with oscillation and rupture after several cycles, (iii) destruction zone with immediate rupture of the shell and release of the gas content.

The shell properties in the previous model can be adjusted to obtain the buckling transition (crosses on figure 7) and the rupture transition (circles) at the same level as experimental transitions to regime (ii) and regime (iii) respectively. The shape of the predicted curves of the critical pressure are in very good agreement with the experimental ones, with the transition parameters set to $\sigma_{m}^{\text {buckling }}=-1 \mathrm{~N} / \mathrm{m}$ and $\sigma_{m}^{\text {break-up }}=3.5 \mathrm{~N} / \mathrm{m}$. The model results are less sensitive on the value of $2 \mathrm{D}$ compression modulus., We nevertheless found an optimum around $\chi_{2 D}=10 \mathrm{~N} / \mathrm{m}$, meaning a relative radius variation of $-5 \%$ at buckling, and $+17.5 \%$ at break-up using Eq. 10, meaning that the linear approximation for the elastic behaviour is still acceptable.

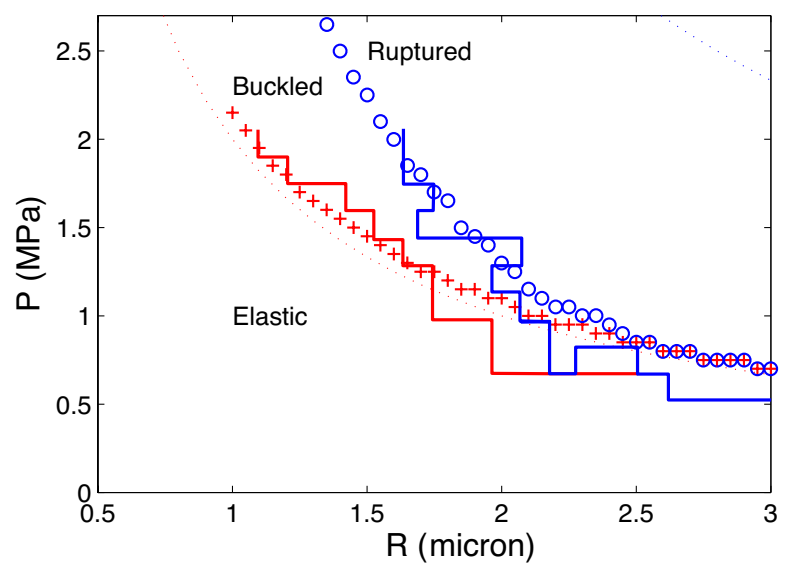

FIG. 7. Phase diagram of the different oscillation behaviour. Thick lines: experimentally measured on polymer contrast agents $^{9}$ boundaries for onset of transient zone (first thick line when increasing pressure) and onset of immediate rupture (second thick line). Symbols: numerical prediction, the shell parameters being adjusted to fit the measured boundaries, with the onset of buckling (crosses) and the onset of rupture (circles). The static buckling pressure $2 \sigma_{m}^{\text {buckling }} / R_{0}$ (first dotted line when increasing pressure) provides a good approximation of buckling, while the static rupture pressure (second dotted line) is overestimating the dynamical value. Shell parameters are $\chi_{2 D}=10 \mathrm{~N} / \mathrm{m}, \kappa_{s}=7.2 \times 10^{-9} \mathrm{~N}$, while the buckling tension is $\sigma_{m}^{\text {buckling }}=-1 \mathrm{~N} / \mathrm{m}$, and the rupture tension is $\sigma_{m}^{\text {break-up }}=3.5 \mathrm{~N} / \mathrm{m}$, under an ultrasound frequency of $f=1.7 \mathrm{MHz}$.

Note that transition from regime (i) to regime (ii) is not experimentally defined as a buckling transition. However, the optical observations reported that buckling allows large shell deformation, that could trigger rupture after several cycles.

We do not model the fatigue of the material that allow rupture after several cycles. The obtained value for the rupture tension thus describes the initial properties of the material, and predicts the immediate rupture only.

\section{B. Wrinkles}

(a)

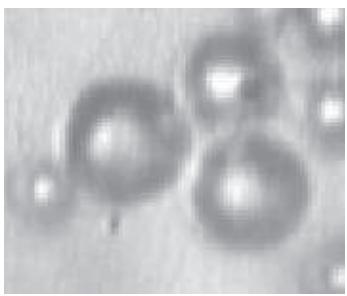

(c)

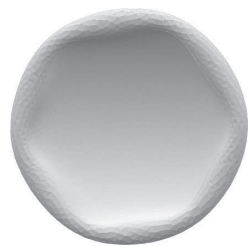

(b)

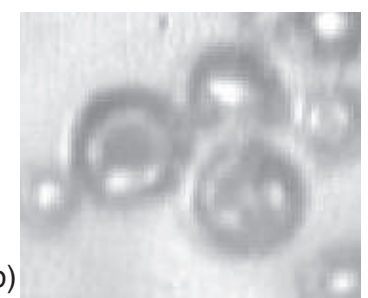

(d)

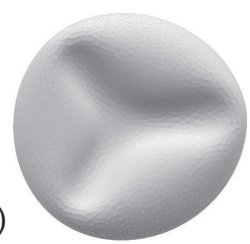

FIG. 8. Top: Snapshots from high-speed recordings of an encapsulated polymer/albumin at two moments during an acoustic cycle: (a) in the inflated state, and (b) in buckled state with wrinkles. Bottom: Numerical simulation of a fully deflated shell $\Delta V / V=0.759$ (c) and a shell under reinflation $\Delta V / V=0.234(\mathrm{~d})$. The shell has a relative thickness $d / R=3 / \sqrt{\gamma}=0.0385$.

A high-speed recording of the shape of the shell previously studied reveals an interesting pattern during the bubble compression (see pictures of figure 8). The recordings were performed with the camera Brandaris ${ }^{31}$, under insonification at $f=1.7 \mathrm{MHz}$, and an acoustic pressure of $0.3 \mathrm{MPa}$ (mechanical index of 0.22 ) for a few cycles. Wrinkles appear around an indentation, which is a materialization of the fact that the surface prefers to fold rather than to compress. The number of wrinkles that can be observed suggests that bubbles hold 6 wrinkles at maximum. Smaller numbers appear during re-inflation.

The comparison with numerical results presented in section II.B.3 indicates, using Eq. 8, a relative thickness in the range $d / R \simeq 0.02$.

\section{Other destruction experiments}

Rupture experiments of solid shells were also performed by $^{32}$ with $100 \mathrm{~nm}$ thick polymeric shells, with a radius $1.45 \mu \mathrm{m}(d / R=0.069)$. For these thin shells it is very likely that they buckle before rupture, and indeed they exhibit a 'nonspherical shape' (quoting the description of ${ }^{32}$ ). The average number of wrinkles associated to 
this shell thickness is 4 according to Eq. 8, a number that could be seen in figure 3 -e of ${ }^{32}$, a statement that should be taken with caution because of the limited resolution.

\section{SUMMARY AND PERSPECTIVES}

In this manuscript, simulations of thin elastic shells showed that the inner pressure difference suddenly drops after buckling and then plateaus, which is a totally unexpected result allowing very practical simplifications when modeling the deflation of elastic spherical surfaces. The amplitude of the drop in pressure and the appearance of wrinkles is quantified.

We translated this behavior in a model with three states, elastic with a finite ability of resistance of shells to compression, buckled above threshold in compression with a vanishing pressure resistance, and finally ruptured when bubble expansion is too large. We expressed these behavior in terms of effective membrane tension rather than pressure, to stress out the shell properties.

The oscillating bubble model provides a threshold in acoustic amplitude for buckling, which can be simply predicted from the static values. The acoustic threshold for rupture occurs at lower amplitudes than expected from the static prediction: indeed bubbles oscillate violently because of periodic buckling, and the inertia helps in creating membrane tensions.

The model can be used to describe the experimental data for the buckling and rupture thresholds. The appearance of wrinkles is a side effect that we also use to have an estimation of the shell thickness: the thinner the shell, the larger the number of wrinkles are to be expected.

Perspectives of this present work include a comparison of the model with other shells with different material properties, in order to confirm the validity of the prediction for the critical buckling pressure. On the theoretical side, a more precise description of $3 \mathrm{D}$ dynamics of the shape during the oscillations would be helpful to set the limits of the approximation of a shape independent dynamic model, where only the bubble volume is modeled.

\section{Acknowledgments}

C.Q. thanks Denis Caillerie for clarifying some features concerning 2D elasticity and Ken Brakke for maintaining Surface Evolver accessible to the scientific community, and tirelessly giving accurate answers to her questions.

\section{APPENDIX A: LINK BETWEEN BULK AND 2D MODEL ELASTIC PARAMETERS}

In the linear approximation, the constitutive relation between the deformation and stress tensors (resp. $\overline{\bar{\varepsilon}}$ and $\overline{\bar{\sigma}}$ ) of an isotropic material is the Hooke's law ${ }^{29}$ :

$$
\overline{\bar{\varepsilon}}=\frac{1}{2 G} \overline{\bar{\sigma}}-\frac{1}{2 G} \frac{\nu}{1+\nu} \operatorname{Tr}(\overline{\bar{\sigma}}) \overline{\bar{I}},
$$

where $G$ is the material's shear modulus, and $\nu$ the Poisson ratio. For a flat thin sheet, e.g. in the $x y$ plane, a deformation is called "longitudinal" when $\sigma_{i z}=0^{29}$. In order to model such a thin sheet by a surface, we may consider the constraint $\overline{\bar{\sigma}}=\left(\begin{array}{ccc}-P_{\|} & 0 & 0 \\ 0 & -P_{\|} & 0 \\ 0 & 0 & 0\end{array}\right)$, acting as an isotropic 2D "pressure", and compare the deformation energy $E_{/ \text {vol. unit. }}=\frac{1}{2} \overline{\bar{\sigma}}: \overline{\bar{\varepsilon}}$ to the $2 \mathrm{D}$ expression: $E_{/ \text {surf.unit }}=\frac{1}{2} \chi_{2 D}\left(\frac{\Delta A}{A}\right)^{2}$. This provides, at first-order, the link between a $2 \mathrm{D}$ and a $3 \mathrm{D}$ description:

$$
\chi_{2 D}=\frac{1+\nu}{1-\nu} G d
$$

where $d$ is the sheet thickness. A similar calculation shows that the equivalent 2D Poisson ratio in such a longitudinal deformation boils down to its three dimensional counterpart:

$$
\nu_{2 D}=\nu
$$

When the normal stresses cannot be neglected any more compared to tangential stresses, a correction $\delta d$ on the thickness $d$ has to be taken into account in this linear model when linking 2D and 3D parameters. One can show that $\delta d=\frac{\nu}{2(1-\nu)} \frac{R_{0}}{G}\left(P_{\text {ext }}-P_{\text {int }}\right)$. The relative error done in equation $\mathrm{A} 1$ by considering the deformation as longitudinal is then $\frac{\delta d}{d}=\nu \frac{d}{R} \frac{\Delta P_{m}^{\text {post-buckling }}}{\Delta P_{m}^{\text {buckling }}}$. With simulations indicating $\Delta P_{m}^{\text {post-buckling }}$ of order $0.1 \Delta P_{m}^{\text {buckling }}$ (cf figure 2) and $\frac{d}{R}=0.04$ as a maximum value, one sees that this correction, of order $1 \%$ in this paper, is negligible.

\section{APPENDIX B: PREDICTION OF PRESSURE AFTER BUCKLING}

After buckling, an inverted cap appears on the shell. From the expression of the energy of a small cap (using eq. (5) and (6) $\mathrm{in}^{26}$ in which we insert $d=3 \sqrt{\frac{\kappa}{\chi_{2 D}}}$ ), and the model ${ }^{26}$ leads to:

$$
\Delta P_{m}^{\text {post-buckling }}=-\frac{3}{4 \sqrt{2}} G\left(\frac{d}{R}\right)^{5 / 2}\left(\frac{\Delta V}{V}\right)^{-1 / 4}
$$

which writes, with $2 \mathrm{D}$ parameters:

$$
\Delta P_{m}^{\text {post-buckling }}=-\left(\frac{3}{2}\right)^{\frac{5}{2}} \frac{\chi_{2 D}^{\frac{1}{4}} \kappa^{\frac{3}{4}}}{R^{\frac{5}{2}}}\left(\frac{\Delta V}{V}\right)^{-1 / 4}
$$

Or, using $G \propto \sqrt{\chi_{2 D}^{3} / \kappa}$ to adimensionalize:

$$
\frac{\Delta P_{m}^{\text {post-buckling }}}{G} \propto\left(\frac{\Delta V}{V}\right)^{-1 / 4} \gamma^{-5 / 4}
$$

On the other hand, it was shown theoretically (resp. numerically) that the $\frac{\Delta V}{V}$ values at buckling scale with a power $\frac{3}{5}$ (resp. 0.55) of $\gamma^{26}$. This leads an expected 
variation of $\frac{\Delta P_{m}^{\text {post-buckling }}}{G}$ that is a power-law of $\frac{d}{R}$ with an exponent between 2.2 and 2.25.

When re-inflating the shell after this buckling, an hysteresis can be observed, compatible with the first-order nature of the deformation, indicated by the pressure discontinuity.

1 S. Qin, C. F. Caskey, and K. W. Ferrara, "Ultrasound contrast microbubbles in imaging and therapy: physical principles and engineering", Phys. Med. Biol. 54, R27 (2009).

2 C. C. Church, "The effects of an elastic solid surface layer on the radial pulsations of gas bubbles", J. Acoust. Soc. Am. 97, 1510-1521 (1995).

${ }^{3}$ K. Sarkar, W. T. Shi, D. Chatterjee, and F. Forsberg, "Characterization of ultrasound contrast microbubbles using in vitro experiments and viscous and viscoelastic interface models for encapsulation", J. Acoust. Soc. Am. 118, 539-550 (2005).

${ }^{4}$ S. M. van der Meer, B. Dollet, M. M. Voormolen, C. T. Chin, A. Bouakaz, N. de Jong, M. Versluis, and D. Lohse, "Microbubble spectroscopy of ultrasound contrast agents", J. Acoust. Soc. Am. 121, 648-656 (2007).

5 A. A. Doinikov and P. A. Dayton, "Maxwell rheological model for lipid-shelled ultrasound microbubble contrast agents", J. Acoust. Soc. Am. 121, 3331-3340 (2007).

6 A. A. Doinikov, J. F. Haac, and P. A. Dayton, "Modeling of nonlinear viscous stress in encapsulating shells of lipidcoated contrast agent microbubbles", Ultrasonics 49, 269275 (2009).

7 S. Paul, A. Katiyar, K. Sarkar, D. Chatterjee, W. T. Shi, and F. Forsberg, "Material characterization of the encapsulation of an ultrasound contrast microbubble and its subharmonic response: Strain-softening interfacial elasticity model", J. Acoust. Soc. Am. 127, 3846-3857 (2010).

8 E. Stride and N. Saffari, "On the destruction of microbubble ultrasound contrast agents", Ultrasound in Med. \& Biol. 29, 563-573 (2003).

9 A. Bouakaz, M. Versluis, and N. de Jong, "High-speed optical observations of contrast agent destruction", Ultrasound in Med. \& Biol. 31, 391-399 (2005).

10 C.-K. Yeh and S.-Y. Su, "Effects of acoustic insonation parameters on ultrasound contrast agent destruction", Ultrasound in Medicine \& Biology 34, 1281-1291 (2008).

11 P. Marmottant, S. van der Meer, M. Emmer, M. Versluis, N. de Jong, S. Hilgenfeldt, and D. Lohse, "A model for large amplitude oscillations of coated bubbles accounting for buckling and rupture", J. Acoust. Soc. Am. 118, 34993505 (2005).

12 N. de Jong, M. Emmer, C. T. Chin, A. Bouakaz, F. Mastik, D. Lohse, and M. Versluis, "compression-only" behavior of phospholipid-coated contrast bubbles", Ultrasound in Medicine \& Biology 33, 653-656 (2007).

13 A. L. Klibanov, "Ultrasound contrast agents: Development of the field and current status", Top. Curr. Chem. 222, 73106 (2002).

14 E. Glynos, V. Koutsos, W. N. McDicken, C. M. Moran, S. D. Pye, J. A. Ross, and V. Sboros, "Nanomechanics of biocompatible hollow thin-shell polymer microspheres", Langmuir 25, 7514-7522 (2009).

15 A. Katiyar and K. Sarkar, "Stability analysis of an encapsulated microbubble against gas diffusion", Journal of Colloid and Interface Science 343, 42-47 (2010).

16 M. A. Borden and M. L. Longo, "Dissolution behavior of lipid monolayer-coated, air-filled microbubbles: Effect of lipid hydrophobic chain length", Langmuir 18, 9225-9233
(2002).

17 E. Lac, D. Barths-Biesel, N. A. Pelekasis, and J. Tsamopoulos, "Spherical capsules in three-dimensional unbounded stokes flows: effect of the membrane constitutive law and onset of buckling", J. Fluid Mech. 516, 303 (2004).

18 T. R. Porter and F. Xie, "Transient myocardial contrast after initial exposure to diagnostic ultrasound pressures with minute doses of intravenously injected microbubbles : Demonstration and potential mechanisms", Circulation 92, 2391-2395 (1995).

19 K. Wei, A. R. Jayaweera, S. Firoozan, A. Linka, D. M. Skyba, and S. Kaul, "Quantification of myocardial blood flow with ultrasound-induced destruction of microbubbles administered as a constant venous infusion", Circulation 97, 473-483 (1998).

${ }^{20}$ C. M. H. Newman and T. Bettinger, "Gene therapy progress and prospects: Ultrasound for gene transfer", Gene Ther 14, 465-475 (2007).

${ }^{21}$ K. Osawa, Y. Okubo, K. Nakao, N. Koyama, and K. Bessho, "Osteoinduction by microbubble-enhanced transcutaneous sonoporation of human bone morphogenetic protein-2", The Journal of Gene Medicine 11, 633641 (2009).

${ }^{22}$ G. M. Lanza, K. D. Wallace, M. J. Scott, W. P. Cacheris, D. R. Abendschein, D. H. Christy, A. M. Sharkey, J. G. Miller, P. J. Gaffney, and S. A. Wickline, "A novel sitetargeted ultrasonic contrast agent with broad biomedical application", Circulation 94, 3334-3340 (1996), [published erratum appears in Circulation 1997 May 20;95(10):2458].

23 J. R. Lindner, P. A. Dayton, M. P. Coggins, K. Ley, J. Song, K. Ferrara, and S. Kaul, "Noninvasive imaging of inflammation by ultrasound detection of phagocytosed microbubbles", Circulation 102, 531-538 (2000).

24 E. Unger, T. Fritz, T. Matsunaga, V. Ramaswami, D. Yellowhair, and G. Wu, "Therapeutic delivery systems related applications.", (1996), US Patent no. 5542935.

25 S. Komura, K. Tamura, and T. Kato, "Buckling of spherical shells adhering onto a rigid substrate", Eur. Phys. J. E 18, 343-358 (2005).

${ }^{26}$ C. Quilliet, C. Zoldesi, C. Riera, A. van Blaaderen, and A. Imhof, "Anisotropic colloids through non-trivial buckling", Eur. Phys. J. E 27, 13-20 (2008), [published erratum appears in Eur. Phys. J. 2010 available at DOI: 10.1140/epje/i2010-10633-0].

27 K. Brakke, "The surface evolver", Exp. Math. 1, 141-165 (1992).

28 J. Lidmar, L. Mirny, and D. R. Nelson, "Virus shapes and buckling transitions in spherical shells", Phys. Rev. E 68, 051910- (2003).

29 Landau and Lifshitz, Theory of Elasticity (Elsevier, Oxford) (1986), 189 pages.

30 N. de Jong and L. Hoff, "Ultrasound scattering properties of albunex microspheres", Ultrasonics 31, 175-181 (1993).

31 C. T. Chin, C. Lancée, J. Borsboom, F. Mastik, M. Frijlink, N. de Jong, M. Versluis, and D. Lohse, "Brandaris 128: A digital 25 million frames per second camera with 128 highly sensitive frames", Rev. Sci. Instrum. 74, 50265034 (2003).

32 S. H. Bloch, M. Wan, P. A. Dayton, and K. W. Ferrara, "Optical observation of lipid- and polymer-shelled ultrasound microbubble contrast agents", Appl. Phys. Lett. 84, 631-633 (2004). 